\title{
How Things Hang Together
}

\author{
George Scialabba
}

\author{
Convergence: The Idea at the Heart of Science \\ by Peter Watson \\ Simon \& Schuster, 543 pp., USD $\$ 35$
}

In Dreams of a Final Theory, Steven Weinberg propounds a familiar but unfailingly stirring claim:

Scientists have discovered many peculiar things, and many beautiful things. But perhaps the most beautiful and the most peculiar thing that they have discovered is the pattern of science itself. Our scientific discoveries are not independent isolated facts; one scientific generalization finds its explanation in another, which is itself explained by yet another. By tracing these arrows of explanation back toward their source we have discovered a striking convergent pattern-perhaps the deepest thing we have yet learned about the universe. ${ }^{1}$

Peter Watson, at any rate, was stirred and has written a lively and colorful volume to illustrate that "convergent pattern." Watson is a prolific novelist and popular historian of art, ideas, and now science. As the titles of his books suggest-e.g., Ideas: A History of Thought and Innovation, from Fire to Freud; The Modern Mind: An Intellectual History of the 20th Century; The Great Divide: Nature and Human Nature in the Old World and the New; The Age of Atheists: How We Have Sought to Live Since the Death of God-Watson is a Big Picture man. Convergence promises a "master narrative" of "synthesis, symphysis, and coherence" among the sciences, in which

the intimate connections between physics and chemistry have been discovered. The same goes for the links between quantum chemistry and molecular biology. Particle physics has been aligned with astronomy and the early history of the evolving universe. Pediatrics has been enriched by the insights of ethology; psychology has been aligned with physics, chemistry, and even with economics. Genetics has been harmonized with linguistics, botany with archaeology, climatology with myth-and so on and so on. ${ }^{2}$

Perhaps depth and originality of insight, or intricacy of argument, are too much to demand alongside such grand sweep and brisk pacing. Convergence will not deeply engage academic philosophers or even historians of science. But it is superior popularization, and very satisfying in its way.

One of the book's strengths is its wealth of anecdote and biographical detail. Watson begins with the poignant story of Mary Somerville, one of those remarkable women who seem to be emerging from the historical shadows with increasing frequency. Somerville was a brilliant, self-taught mathematician who was published in the Proceedings of the Royal Society and numbered dozens of its fellows among her friends, although women were not allowed to attend lectures at the Society until after her death. Her second book, On the Connexion of the Physical Sciences (1834), one of the first to trace a pattern of unification and simplification in the discovery of physical laws, was reprinted throughout Europe and praised by James Clerk Maxwell.

Perhaps the most discerning review of Somerville's Connexion was by the historian and philosopher of science William Whewell, who pointed out that until then, most commentators on science had been struck rather by its increasing divergence and diversity. There was not even an agreed name for workers in the field-Whewell coined the term "scientist" (and later "physicist" and "consilience"). But his and Somerville's perception of the advancing unification of the sciences was vindicated, Watson writes, by the emergence in the 1850s of "the two most powerful unifying theories of all time": the conservation of energy, and biological evolution. ${ }^{3}$

Watson recounts the early experiments of Michael Faraday, James Prescott Joule, Julius Lothar Meyer, and William Thomson, Lord Kelvin on heat, electricity, and magnetism, summarized as the laws of thermodynamics in papers by Hermann von Helmholtz in 1847 and Rudolf Clausius in 1850. Following this, Maxwell and Ludwig Boltzmann introduced statistics into thermodynamics, allowing the velocities, spatial distribution, and collision probabilities of the molecules in a gas to be calculated and introducing the concept of entropy as the measure of the order of a system. 
(In passing, Watson takes note of Thomas Kuhn's suggestion that the conservation of energy was suggested to its German pioneers by the writings of Friedrich Schelling and other Naturphilosophen, who maintained that "magnetic, electrical, chemical, and finally even organic phenomena would be interwoven into one great association." ${ }^{4}$ If true, this would seem to reverse the usual direction of influence between science and philosophy.)

Charles Darwin's career and the publication of On the Origin of Species might seem so well documented that even a resourceful popular historian would be at pains to narrate them interestingly. But Watson rises to the challenge with an account of developments in two apparently unrelated fields, which nonetheless were indispensable parts of the Origin's intellectual background. William Herschel's astronomical observations, including his discovery of Uranus and of hundreds of nebulae, and above all his theories of galactic formation and evolution, changed the character of astronomy "from a mathematical science concerned primarily with navigation, to a cosmological science concerned with the evolution of stars and the origins of the universe." ${ }^{5}$ (And once again Watson draws a remarkable woman out of the shadows-Herschel's sister Caroline, who served as his assistant and herself discovered eight comets.)

The other science in the background of evolution was geology, its story much better known. Watson traces its development through its early practitioners-William Buckland, Georges Cuvier, James Hutton, Roderick Murchison, Adam Sedgwick-to the discovery of the Ice Age by Louis Agassiz and, most familiarly, to Charles Lyell, whose Principles of Geology made an irrefutable case for the Earth's being far older than previously assumed. Without these crucial advances in cosmology and geology, Watson writes, "Darwin would not have been plausible." ${ }^{6}$

The next great unification was that of physics and chemistry. The discovery of the periodic table of the elements in the late 1860s, Watson observes, "gave chemistry a central idea to put alongside [Isaac] Newton's in physics and Darwin's in biology." In subsequent decades, Henri Becquerel, Heinrich Hertz, Wilhelm Röntgen, and other physicists, experimenting with electromagnetism, discovered the phenomenon of radioactivity. X-rays were immediately added to the arsenal of medicine, while radium, radon, polonium, and other elements were added to the periodic table. In 1911 Ernest Rutherford's bombardment of metal foil with beams of electrons revealed the planetary structure of atoms, with electrons orbiting around a positively charged nucleus.

All these strands were drawn together in Niels Bohr's celebrated trio of papers, "On the Constitution of Atoms and Molecules." It was not clear why, in Rutherford's model, the orbiting electrons did not either fly apart or collapse into the nucleus. Bohr recognized that the quantum nature of matter meant that only certain orbits were permissible. This insight, that "although the radioactive properties of matter originate in the atomic nucleus, the chemical properties reflect primarily the distribution of electrons," explained "at a stroke ... the link between physics and chemistry." ${ }^{\circ}$ Within a decade, Bohr had married the two fields even more closely by explaining the similar properties of each family of elements in the periodic table in terms of the arrangement of electrons in their outermost orbit, an achievement Albert Einstein delightedly described as "the highest form of musicality in the sphere of thought." 9

Linus Pauling is, along with Bohr, one of the heroic unifiers in Watson's account. The nature of the chemical bond was the theoretical holy grail among early twentieth-century chemists. Because Pauling knew far more about crystallography and quantum theory than most chemists and more about chemical properties than most physicists, he could

distill what he knew about quantum mechanics, ionic sizes, and crystal structures, and put that together with a traditional understanding of the habits of the elements, all wrapped up into a set of rules for indicating which "joining patterns" were most likely. ${ }^{10}$

With his further discovery of "resonance"-the coexistence of ionic and covalent bonds between atoms in a single molecule-Pauling was able to explain the tetrahedral bonding of carbon atoms, the puzzling reactivity of benzene, and the structure of more than two hundred other, mostly organic, molecules, in effect birthing the science of molecular biology.

The next phase of the "friendly invasion of the biological sciences by the physical sciences," Watson writes, came via Erwin Schrödinger's What Is Life? which looked at heredity from the physicist's point of view. Schrodinger estimated the dimensions and structure of the chromosome, and was the first to characterize it as "a message written in code."11 According to Watson, What Is Life? deeply influenced DNA researchers Francis Crick, James Watson, and Maurice Wilkins, as well as the equally important work on protein structure in the 1950s.

In the 1960s and 1970s, physics experienced its own internal consolidation. The discovery of the cosmic background radiation, of the subatomic particles found in cosmic rays, and of quasars and pulsars were "all synthesized into one consistent, coherent, unified story, to produce a detailed assessment about the origin and evolution of the universe." ${ }^{2}$ Watson calls it "the second evolutionary synthesis."13

The last third of Convergence is at once the most interesting and the least readily assimilated to Watson's grand narrative of the unification of the sciences. It mostly (apart from somewhat breathless overviews of information theory, string theory, and many universes theory) deals 
with recent developments in planetary science and social science. Just as cosmology, in working out the biography of the universe, depended on advances in particle physics, paleontology, in writing the biography of the earth, required new microphysical tools and techniques, above all radioactive dating based on the half-lives of uranium and carbon.

The moon landing also helped, Watson suggests, to solve a key paleontological puzzle: the K-T boundary, or the exceptionally sharp divide in the fossil record between the Cretaceous and Tertiary periods 65 million years ago. The frequency of cratering on the moon led to speculation that an asteroid had caused a large-scale extinction on earth. Physicists helpfully pointed out that impact sites would be rich in iridium, which is absorbed in naturally occurring rocks by the earth's iron core. Iridium, according to Watson, was the key clue that led to the discovery of the Yucatan crater where the great asteroid hit. It is undeniable in this case that physics expanded the paleontologist's toolkit. Whether that amounts to a unification of the two sciences is another matter.

Watson's chapter on "Big History" is even more interesting. Carefully and imaginatively, he traces several lines of evidence, including myths, archaeological artifacts, paleogenetics, linguistics, and astronomy, to deduce a convincing story of the origins and early migrations of Homo sapiens. His surprising (to me, at least) conclusion is that "for approximately 16,500 years-from 15,000 $\mathrm{BC}$ to $\mathrm{AD} 1,500,640$ generations-there were two populations of people in the world who, insofar as we know, were unaware of each other."14 In other words, civilization evolved twice.

By contrast, and a little anticlimactically, a chapter on sociobiology and evolution covers mostly familiar ground. It falls short, at any rate, of establishing Watson's claim that Jacques Monod's Chance and Necessity (1970) and Edward O. Wilson's Sociobiology (1975) mark "the watershed moment when the coming together-the convergence-of the sciences achieves such resonance that science itself becomes the basis for comprehending other forms of knowledge. [emphasis original]" 15

$\mathrm{S}$ OME AUTHORS ARE storytellers; others make arguments. Few authors, I suspect, are equally skilled and comfortable at both. Even among storytellers, there is a distinction among dramatists of personality and dramatists of ideas. The best intellectual history makes ideas into characters, whose biography-birth, maturity, decline-engages us even as their adherents' lives and circumstances seem incidental. Watson is not this kind of historian, able to give his story something like sonata form, with a leading theme followed by its development, abstract and sensuous at the same time. Nor is he particularly rigorous; his idea of convergence is a little loose and baggy, almost promiscuously inclusive, with mere connec- tion or analogy sometimes standing in for unification. It seems a bit cavalier, for example, to claim D'Arcy Thompson as a prophet of unification for maintaining that natural selection "cannot by itself possibly account for the diversity we see around us" but instead must have been "aided by the self-organization of matter based on mathematical and physical principles."16 Wouldn't that make Thompson a complicator rather than a unifier?

Watson is instead a fluent and enthusiastic personalizer, quick to drop the thread of conceptual continuity in order to relay an anecdote or display a piquant quote. Fortunately, most of his anecdotes and quotes are well-judged. It is amusing, for example, to learn that Einstein's Greek teacher informed him that "whatever field in life he chose, he would fail at it,"17 likewise, to hear about J. J. Thomson, director of the Cavendish Laboratory, that

one day he bought a pair of new trousers on his way home for lunch, having been convinced by a colleague that his old pants were too baggy and worn. At home he changed into his new trousers and returned to the lab. His wife, arriving home from a shopping trip, found the worn-out pair on the bed. Alarmed, she hurriedly telephoned the Cavendish, convinced that her somewhat absentminded husband had gone back to work without any trousers on. ${ }^{18}$

In a different vein, it is poignant to overhear the troubled Wolfgang Pauli confessing his predilection for the Viennese "night, sexual excitement in the underworldwithout feeling, without love, indeed without humanity."19 And the book's account of nuclear physicist Lise Meitner's escape from the Nazis is thrilling.

But philosophical questions are not ignored in Convergence, even if they are not pursued with the depth and precision they might have been. Watson devotes a chapter to the Unity of Science movement in the 1930s, discussing several contributions to the first edition of the International Encyclopedia of Unified Science (1938). Because the protagonists of the movement were the logical positivists of the Vienna circle, the question of physicalism was central. But the nature and complexities of that doctrine, and its subsequent vicissitudes in the philosophy of science, are barely acknowledged.

There is, however, in a later chapter, a long discussion of an important paper from the 1950 s by Hilary Putnam and Paul Oppenheim, "Unity of Science as a Working Hypothesis." The paper listed six "reductive levels," in descending order: social groups, multicellular organisms, cells, molecules, atoms, and elementary particles. That all these "may one day be reduced to microphysics (in the sense in which chemistry seems today to be reduced to it)" ${ }^{20}$ seemed to them a reasonable expectation. But they closed on a more equivocal note, with a quote from the general systems theorist Ludwig von Bertalanffy, which instead of strict reductionism spoke of 
a superposition of many levels, from physical and chemical to biological and sociological systems. Unity of Science is granted, not by any utopian reduction of all sciences to physics and chemistry, but by the structural uniformities of the different levels of reality. ${ }^{21}$

In the book's final chapter, "A Pre-Existing Order?", dissenting voices are heard from. The leading theme of the opposition to reductionism is emergence: the observation that, at a certain level of complexity, new properties sometimes appear that cannot be explained or predicted by the known rules of interaction among the smaller units involved. Life and consciousness are the most commonly cited examples, though Watson also mentions "processes of self-organization leading to nonhomogenous structures and nonequilibrium crystals." 22 In these cases, "microscopic rules can be perfectly true and yet quite irrelevant to [the resultant] macroscopic phenomena." ${ }^{23}$ As Robert Laughlin, a prominent critic of reductionism, puts it:

The laws of nature that we care about ... emerge through collective self-organization and really do not require knowledge of their component parts to be comprehended and exploited. ... [P] hysical science [has] stepped firmly out of the age of reductionism into the age of emergence. The shift is usually described in the popular press as the transition from the age of physics to the age of biology, but that is not quite right. What we are seeing is a transformation of a worldview in which the objective of understanding nature by breaking it down into ever smaller parts is supplanted by the objective of understanding how nature organizes herself. $^{24}$

Does emergence undermine convergence? Watson cheerfully shrugs off the challenge:

The story told in these pages is not a straight line ... but it is a line, a narrative, which hangs together, and is not a mere artifact of the instruments with which the observations have been carried out. There is an order to our world, and how we got here..$^{25}$

Others are less confident. To the physicist John Barrow, "extremes of complexity ... reveal the limits of a reductionism that looks to a Theory of Everything to explain the totality of the natural world from the bottom to the top." Reductionism may be "trivially true," in that it helps us eliminate metaphysical mysteries like the élan vital. But complex aggregates display "a wider diversity of behavior than the sum of their parts," so that "if reductionism means that all explanations of complexity must be sought at a lower level, and ultimately in the world of the most elementary constituents of matter, then reductionism is false." ${ }^{26}$ The astrophysicist John Gribbin, surveying the same phenomena, comes to an apparently opposite conclusion:

[C]haos and complexity obey simple laws-essentially, the same simple laws discovered by Isaac Newton more than three hundred years ago. Far from overturning four centuries of scientific endeavor as some accounts would lead you to believe, these new developments show how the long-established scientific understanding of simple laws can explain (although not predict) the seemingly inexplicable behavior of weather systems, stock markets, earthquakes, and even people. ... [T] ]he complicated behavior of the world we see around us ... is merely "surface complexity arising out of deep simplicity."27

I say "apparently" opposite because the ground of the disagreement-the meaning of "reductionism"-is not altogether clear. Clearly the Standard Theory of elementary particles does not explain consciousness or even protein structure. But just as clearly, no one claims that it does. What is often claimed, rather, is that theories of simpler forms of matter underlie theories of more complex forms. "Underlie" is a metaphor, and so needs to be unpacked. Perhaps "constrain," in the sense of "limit," is the operative meaning. That is, a lower-level theory (e.g. the theory of elementary particles) constrains a higher-level theory (e.g. the theory of protein structure) in the sense that, if both are well established, and are incompatible, the higher-level theory must give way. Then again, what would that mean? In practice, an incompatibility of that sort would simply motivate redoubled efforts to confirm that the two theories were well established and that they were genuinely incompatible. And if so, the only reasonable attitude would be a temporary suspension of judgment, uncomfortable though that might be.

It may be, as Frank Wilczek writes, that "reductionism has a bad name ... because 'reductionism' is a bad name." It suggests a blinkered "no more than"-ism, rather than, as Wilczek and his fellow Theorists of Everything experience it, "a spiritual quest, reaching for the sublime." 28

Spiritual quests do not always end well, of course. The presiding genius of Convergence is Einstein, who avowed in his Nobel Prize lecture that "the mind striving for unification cannot be satisfied that two fields should exist which, by their nature, are quite independent." ${ }^{29}$ A stirring sentiment; but, as Watson acknowledges, Einstein died unsatisfied.

George Scialabba is a contributing editor of the online art and literary magazine The Baffler.

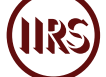


1. Steven Weinberg, Dreams of a Final Theory (New York: Pantheon Books, 1992), 19.

2. Peter Watson, Convergence: The Idea at the Heart of Science (London: Simon \& Schuster, 2016), xxiii, xxii.

3. Watson, Convergence, 16.

4. Watson, Convergence, 22.

5. Watson, Convergence, 52.

6. Watson, Convergence, 54.

7. Watson, Convergence, 85.

8. Watson, Convergence, 102.

9. Watson, Convergence, 128.

10. Watson, Convergence, 146.

11. Watson, Convergence, 204, 220

12. Watson, Convergence, 256.

13. Watson, Convergence, 245.

14. Watson, Convergence, 294.

15. Watson, Convergence, 313.

16. Watson, Convergence, 442.
17. Watson, Convergence, 106.

18. Watson, Convergence, 93.

19. Watson, Convergence, 129.

20. Watson, Convergence, 238.

21. Watson, Convergence, 238.

22. Watson, Convergence, 436.

23. Watson, Convergence, 480.

24. Watson, Convergence, 480-81.

25. Watson, Convergence, 487.

26. John Barrow, New Theories of Everything: The Quest for Ultimate Explanation (Oxford: Oxford University Press, 2007), 160, 164.

27. John Gribbin, Deep Simplicity: Bringing Order to Chaos and Complexity (New York: Random House, 2004), xxiiiii.

28. Frank Wilczek, A Beautiful Question: Finding Nature's Deep Design (New York: Penguin, 2014), 112, 114.

29. Watson, Convergence, 122-23. 